OPEN ACCESS

Edited by:

Feng Jiang,

Central University of Finance

and Economics, China

Reviewed by:

Weipeng Lin,

Shandong University, China

Guiquan Li,

Rennes School of Business, France

${ }^{*}$ Correspondence:

Xiaopeng Ren

renxp@psych.ac.cn

Specialty section:

This article was submitted to

Cultural Psychology,

a section of the journal

Frontiers in Psychology

Received: 28 August 2020

Accepted: 29 March 2021

Published: 13 May 2021

Citation:

He L, Chen Y and Ren X (2021) Climato-Economic Origins

of Variations in Uniqueness

of Nickname on Sina Weibo.

Front. Psychol. 12:599750.

doi: 10.3389/fpsyg.2021.599750

\section{Climato-Economic Origins of Variations in Uniqueness of Nickname on Sina Weibo}

\author{
Lingnan $\mathrm{He}^{1}$, Yue Chen ${ }^{1}$ and Xiaopeng Ren ${ }^{2 *}$ \\ ${ }^{1}$ School of Communication and Design, Sun Yat-sen University, Guangzhou, China, ${ }^{2}$ Institute of Psychology, Chinese \\ Academy of Sciences, Beijing, China
}

In the world of social media, people are free to choose names based on their preferences, which may potentially reflect certain levels of uniqueness. In this study, we have attempted to explore the possibility of applying the ecological theory of individualism/collectivism in the context of social media. We, thus, examined provincial variations in the uniqueness of nicknames among more than 13 million Sina Weibo users. Initially, the nickname uniqueness indicator was set at the provincial level. It was found that the uniqueness of nicknames was the highest in provinces with temperate climates, for example Guangdong, and the lowest in provinces with demanding climate, such as Ningxia. Regression analysis results partially supported that inhabitants in provinces with temperate climate were more likely to use unique nicknames on social media compared to those from harsh climate. This finding is significant in terms of ecology.

Keywords: uniqueness, climate demand, social ecology, social media network, China

\section{INTRODUCTION}

Billions of people all over the world currently have their profiles in online social networks (OSNs). OSNs are widely used as the primary medium for communication and networking. Users often use nicknames, rather than their real names, as identifiers to be found and remembered by others, as well as to express their preference. Cultural studies on real names found that the uniqueness of real names at the group level is an indicator of individualism and functions as a social-ecological factor (Twenge et al., 2010; Varnum and Kitayama, 2011).

Does it also work for nicknames? Some authors have argued that people on online social media platforms behave in a similar manner to real settings and function as if it is real life (Back et al., 2010). This suggests that inhabitants in an individualistic culture or region would be inclined to pick unique nicknames for themselves than their counterparts in a collectivistic one. However, there is little research on the subject. 
Measurable variations of collectivism have been proven within Mainland China (Hou et al., 2016; Stojcic et al., 2020). Some socio-ecological factors were found to predict provincial variations of collectivism, such as climate demand (Van de Vliert et al., 2013) and herder-wheat-rice farming (Talhelm et al., 2014; Stojcic et al., 2020). Sina Weibo has 516 million users all over China (Statista, 2020). Millions of users' nicknames could be used to measure their uniqueness. Therefore, we tried to examine whether there are provincial differences in the uniqueness of nicknames and its relationship with climate demand.

\section{UNIQUENESS}

Uniqueness is a key facet of individualism/collectivism (IC) (Oyserman et al., 2002). I-C may be the most important dimension that captures cultural differences in human behavior (Oyserman, 2017). Compared to an individualistic culture, people in a collectivistic one are more embedded in their tight social networks and care more about relational or group harmony. I-C has been proven to be a loosely connected construct, in which the different facets or measurements are weakly related to each other ( $\mathrm{Na}$ et al., 2020). People in an individualistic culture prefer to differ from others or stand out from groups than those in a collectivistic one. In a content analysis of 27 individualism/collectivism scales, personal uniqueness was identified as one of seven facets of individualism, in which about $30 \%$ of the scales included items measuring personal uniqueness, such as "I'm different from others in many respects." Furthermore, in the follow-up meta-analysis, uniqueness was found to be sensitive to cultural differences (Oyserman et al., 2002). Kim and Markus found that European Americans would select pens with unique colors as presents as opposed to Asian Americans, as the differently colored pens indicated their uniqueness (Kim and Markus, 1999). When naming babies, people are more inclined to use unique names in an individualistic culture or regions than those in a collectivistic culture. As a result, the percentage of the most common first names has been constructed as an indicator of I-C, where a higher percentage of the most common first name means higher collectivism or less individualism for a certain group. For example, a study that used data from 13 nations, which included nine European nations and four frontier nations (United States, Canada, Australia, and New Zealand), found that the percentage of the most common first names was negatively correlated to Hofstede's individualism (Varnum and Kitayama, 2011). Furthermore, along with the increase in individualism in the United States, people had started giving babies unique names, which resulted in a decrease in the percentage of the most common first names (Twenge et al., 2010).

The link between uniqueness and I-C exists not only in intercultural comparisons but also in intracultural regional comparisons. Furthermore, intracultural comparisons would give an additional validity for controlling the more confounding factors such as language, religion, etc. (Liu et al., 2019). In Varnum's studies 1 and 2, the percentages of the most common baby names were higher in less individualistic regions (eastern regions) than in the more individualistic ones (western frontier regions) in both the United States and Canada separately (Varnum and Kitayama, 2011). Others found that frontier experiences during 1790-1890 were associated with giving babies uncommon names at the county level in the United States (Bazzi et al., 2020). Overall, fitting in or standing out from a group in the practice of naming babies has been strongly proven to be a facet of I-C.

\section{CLIMATO-ECONOMIC HYPOTHESIS OF UNIQUENESS}

In general, uniqueness is an outcome of psycho-behavioral adaptation toward a climate demand and the income resource of inhabitants' environment. Derived from the demands and resources theory, the climato-economic theory of culture posits that culture is shared psycho-behavioral adaptations toward the interaction of climate demands and economic resources (Van de Vliert, 2013, pp. 467-469). Since a temperate climatic condition is undemanding and comforting, its inhabitants do not need much resources to survive; thus they make easygoing goals a priority over survival goals. They then like to adopt a convenient agency to deal with problems. They would neither feel pressure to follow the group norms and fit in nor have a strong motivation to stand out from a group when picking up a nickname. Compared with those in regions with moderate climates, inhabitants in regions with extreme summers and harsh winters need more resources to sustain life, such as housing, clothes, and food, in order to cope with the climate stress. Whether inhabitants appraise climate demand to be either threatening or challenging is moderated by the existing resources to cope with it. In hotter-than- and colder-than-temperate climate conditions with poor resources, inhabitants would evaluate it as a threatening condition, which makes them prioritize survival goals over easier goals. They then would prefer to adopt an in-group agency to deal with problems. This implies that inhabitants would be prone to sacrifice some individual freedom to obtain support and resources from groups in order to survive. They are sensitive to group norms and are inclined to conform rather than to stand out in order to maintain group harmony. As a result, inhabitants would be riskaverse and like to pick a common nickname that fits in the group rather than a unique one that stands out. When in hotterthan- and colder-than-temperate climate conditions with rich resources, inhabitants would deem it as a challenging condition and prioritize self-expression over others. Furthermore, they would be likely to adopt an individualistic agency to deal with problems. They would be motivated to be confident and prefer to be self-reliant rather than be dependent on groups for resources to survive. Consequently, inhabitants would be likely to pick a unique name that probably stands out from the group and fits their self-expression goals and the norm of "not following the social norms" rather than picking a common nickname that fits in the group and the norm of "following the social norms."

Although there is no direct evidence of a relationship between climato-economic conditions and the uniqueness of naming 
practice, there is empirical evidence that supports the climatoeconomic hypothesis of I-C. For example, using World Value Survey data, it was found that members of societies in more demanding climate patterns endorse survival values at the expense of self-expression to the extent that the household incomes in these lower-income societies are lower (Van de Vliert, 2007). The interaction of climate demand and income resources also predicted compatriotism, nepotism, and familism (Van de Vliert, 2011), freedoms (Van de Vliert, 2013), overall subjective ill-being composed of health complaints and anxiety and burnout (Fischer and Van de Vliert, 2011), and in-group/outgroup differentiation (Van de Vliert, 2020). Thus, it can be suggested that the climato-economic theory of collectivism could predict uniqueness in naming practice.

\section{USERNAME ON ONLINE SOCIAL MEDIA}

Numerous individuals across the world have their personal profiles on social media, where they engage in different kinds of activities. Usernames, instead of their real names, are often used as labels or markers to be easily found by other individuals or organizations. Usernames are also a convenient way to express the owner's preferences, attitudes, emotions, and so on, especially so for the Chinese as they have a long history of assigning certain connotations or implications to names. Furthermore, unlike a real name, a nickname is usually given by the user himself/herself rather than by his/her parents, which means that the nickname is more closely linked to the individualistic or independent value of the user compared to the real name. Thus, the most common username at group level can be used as an indicator of I-C, following the same mechanism as mentioned before.

\section{CHINA: A NATURAL CASE}

China has an advantage in exploring the relationship between climate demand and the uniqueness of usernames. Firstly, provincial variations of I-C have been found in Mainland China, such as nepotism, thinking style, implicit individualism (Talhelm et al., 2014), composited collectivism (Van de Vliert et al., 2013), and cultural tightness-looseness (Chua et al., 2019). Secondly, ecological factors differ from each other to the extent that they are similar to the national differences in Europe (Ren et al., 2021). For example, the climate demand in Heilongjiang (91.3), which is located in northern China, is similar to that in Norway (89), while those in Hainan (22.9), which is located in southern China, is similar to that in Cook Island (28) (Van de Vliert, 2013; Van de Vliert et al., 2013). Thirdly, the interaction of climate demand and income resource could predict collectivism at the provincial level within the Chinese mainland using a sample from 15 provinces (Van de Vliert et al., 2013). Lastly, the uniqueness of a name, which is made up of the percentage of the most common or popular names (reversed), has been found to support I-C ethnic or regional differences within Mainland China and their relationship with ecological factors such as herding vs. farming (Stojcic et al., 2020) and voluntary frontier settlement (Chen et al., 2019).

The shortcomings of China should also be addressed to explore the effects of climato-economic factors. Firstly, most Chinese provinces have demanding climate conditions (Van de Vliert et al., 2013). Secondly, China is an underdeveloped nation with poor resources from a global perspective. For instance, China's per capita gross domestic product (GDP) ranked 70th worldwide in 2019 (World Bank, 2020). Thus, the overall livability in China would be appraised as threatening rather than challenging (Van de Vliert et al., 2013). Variations of climatoeconomics would not be large enough so that their effects on uniqueness are mild or medium.

Sina Weibo, often referred to as Chinese Twitter, is one of the most influential social networking platforms in China. Over 350 million users are registered on Sina Weibo. Each day, around 130 million words and 1.5 million videos and live streaming are published on the platform (Statista, 2020). Nicknames and location information of registered users could be found publicly on their home page. This is illustrated by a screenshot of a user from this platform (see Figure 1), with a nickname "wan lai tian yi xue" (in Chinese, “晚来天已雪”) and located in Guangdong. They gave us a chance to explore whether or not there are regional variances of name preferences and its relationship with climate demand.

Unfortunately, the percentages of the most common names that had been used before do not work for the usernames on the Sina Weibo platform. When one username such as "renxiaopeng" was used, a new user cannot use this username again on the platform. He/she would be forced to create a new username. It is impossible to use the previous method to calculate the percentages of the most common usernames. Thus, we strived to find a new method to construct the uniqueness of usernames so that regional differences of uniqueness within China can be measured.

Therefore, the aims of the current study were to: (1) create an index of uniqueness based on Sina Weibo users' nicknames at the provincial level and (2) test whether or not the climato-economic theory of collectivism could predict the uniqueness of nicknames at the provincial level in China. We hypothesized that the uniqueness of nicknames would be lowest in provinces with a harsh climate and lower incomes, moderate in those with temperate climate irrespective of income, and highest in those with a harsh climate and higher incomes.

\section{Ethics Statement}

This study involving human participants was reviewed and approved by the Institutional Review Board of the Institute of Psychology, Chinese Academy of Sciences. Written informed consent from the participants was not required to participate in this study, in accordance with the national legislation and the institutional requirements, because our data were collected from publicly available information on an open online social networking site through Weibo's application programming interface. 


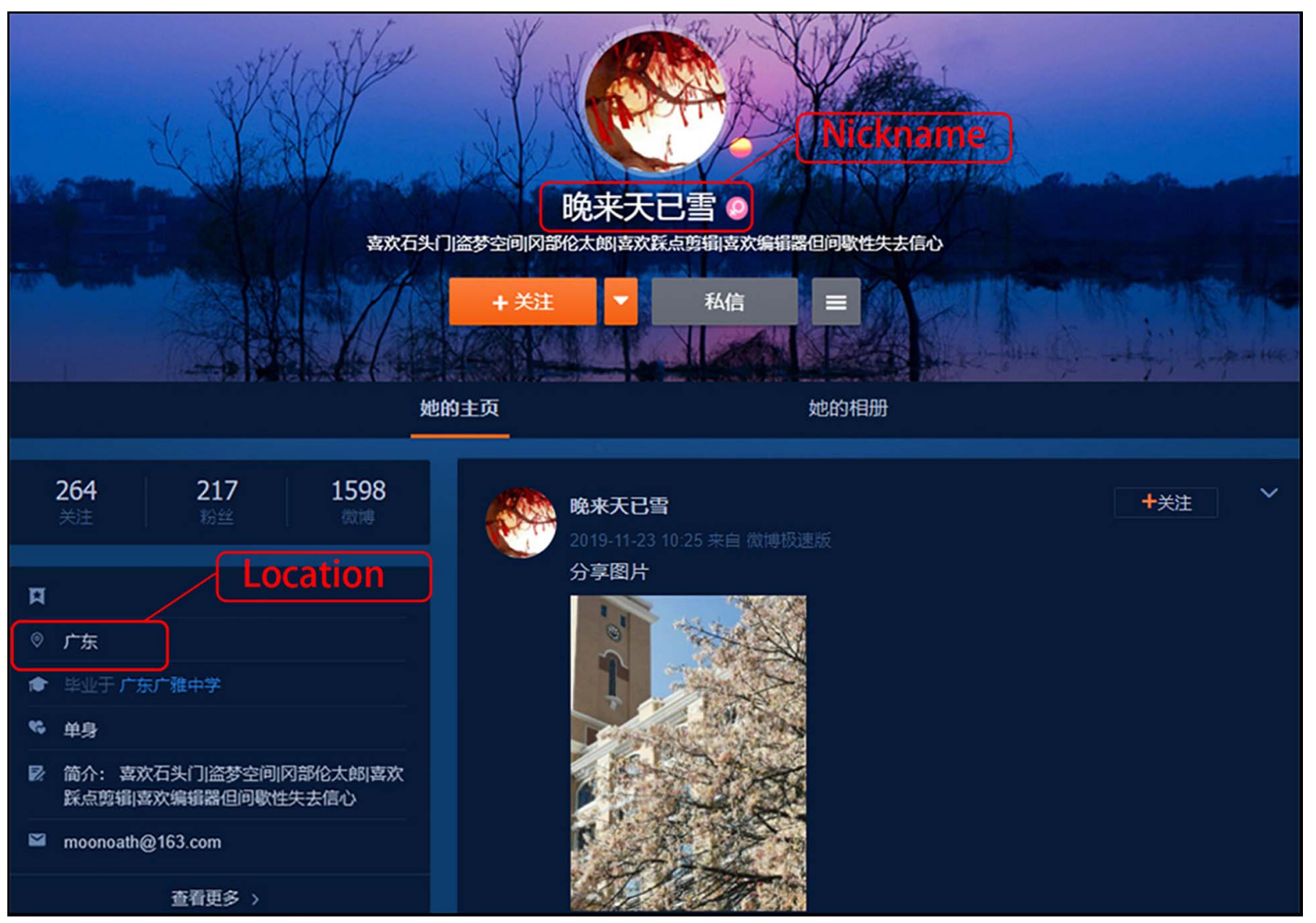

FIGURE 1 | Web page of a Sina Weibo user with the nickname and location.

\section{PART 1: CREATING THE CHINESE UNIQUENESS SCALE}

\section{Methods}

Following Vandello and Cohen's and Yamawaki's strategy in creating a collectivistic scale (Vandello and Cohen, 1999; Yamawaki, 2012), we created the Chinese uniqueness scale at the provincial level (similar to states in the United States and prefectures in Japan) using Sina Weibo users' nicknames. Based on characters of the users' nicknames, we selected a few indicators that we thought were theoretically related to uniqueness. Thereafter, individual items would be moderately correlated to yield a scale with high reliability. These items would have to be non-redundant to fit the parsimonious principle. Finally, a few items were deleted, which will be discussed later. Thus, three items were retained on the Chinese uniqueness scale.

Generally, a user's nickname is quite different from his/her real name. Each Chinese real name is composed of a surname and a first name, in which the surname is written before the first name, for example, Ren Xiaopeng (in Chinese, “任孝鹏”). The first name usually includes one or two Chinese characters. NonChinese characters, such as an Arabic number and an English alphabet, are excluded. However, all these rules are set aside for nicknames. A nickname with far more than three characters or with English alphabets and Arabic numbers can be found easily. This deviation from the rules of real names in China implied constructing uniqueness based on nicknames.

\section{Percentage of Users With Over 10 Chinese Characters in Their Nickname}

As mentioned in the paragraph above, real names mostly comprise two or three Chinese characters, for example, Ren Xiaopeng (in Chinese, “任孝鹏”) or Su Hong (in Chinese, “苏红”). For a nickname, it is also common for users to use four to seven Chinese characters to create a nickname to express his/her uniqueness, for example, "na dou bu shi shi" (in Chinese, “那都不是事”). However, compared with common nicknames, those with over 10 items are infrequent, such as "meng meng xiao jie shi ge ao jiao de xiao gu liang" (in Chinese, “萌萌小姐是个傲娇的小菇凉”). Ten Chinese characters can be considered to be more unique than most nicknames. Thus, having a nickname with over 10 Chinese characters was used as the inclusion criterion for this study.

\section{Percentage of Users With Non-chinese Characters in Their Nickname}

Non-Chinese characters include English alphabets or Arabic numbers or other symbols, such as “*." For example, the nickname "NorthToFace" was thought to be unique. 
TABLE 1 | Provincial ranking on Chinese uniqueness scale index.

\begin{tabular}{|c|c|c|c|c|c|c|c|}
\hline Rank & Province & Score & Nswu & Rank & Province & Score & Nswu \\
\hline 1 & Ningxia & 28 & 62456 & 17 & Yunnan & 45 & 221916 \\
\hline 2 & Tibet & 31 & 39718 & 18 & Heilongjiang & 46 & 266395 \\
\hline 3 & Qinghai & 32 & 48843 & 19 & Zhejiang & 48 & 758421 \\
\hline 4 & Jiangxi & 36 & 243245 & 20 & Jiangsu & 50 & 941354 \\
\hline 5 & Anhui & 37 & 379311 & 21 & Guizhou & 52 & 143428 \\
\hline 6 & Hebei & 38 & 443475 & 22 & Shaanxi & 52 & 351092 \\
\hline 7 & Henan & 38 & 601851 & 23 & Inner Mongolia & 54 & 185780 \\
\hline 8 & Shandong & 40 & 759389 & 24 & Hubei & 55 & 466695 \\
\hline 9 & Hainan & 42 & 91762 & 25 & Sichuan & 55 & 598154 \\
\hline 10 & Fujian & 43 & 459472 & 26 & Chongqing & 55 & 287059 \\
\hline 11 & Guangxi & 43 & 250784 & 27 & Tianjin & 57 & 369552 \\
\hline 12 & Liaoning & 43 & 432508 & 28 & Beijing & 59 & 1119240 \\
\hline 13 & Gansu & 44 & 137517 & 29 & Shanghai & 94 & 779648 \\
\hline 14 & Jilin & 44 & 205736 & 30 & Guangdong & 95 & 1682967 \\
\hline 15 & Hunan & 45 & 355395 & 31 & Xinjiang & 102 & 161522 \\
\hline 16 & Shanxi & 45 & 261719 & & & & \\
\hline
\end{tabular}

Nswu, number of Sina weibo's users.

\section{Percentage of Users With Nicknames With No More Than Five Full Chinese Characters (Reversed)}

Nicknames with all Chinese characters and no more than five full Chinese characters will be similar to a real name, for example, “qing jing ben ran” (in Chinese, “清净本然”). It sounds reasonable to be a common nickname. Therefore, it was reversed to make it unique.

\section{Results}

A total of 16 million Sina Weibo users' posts were randomly extracted from January 1 to December 31, 2017 through Weibo's application programming interface. Users without location information were excluded. Over 13 million users' nicknames from Mainland China were selected for analysis. Users were unevenly distributed all over Mainland China. There are 1,682,967 users in Guangdong Province, while only 39,718 users in Tibet. This are listed in Table 1. All three indicators were calculated on the users' nicknames.

We calculated the indicators of uniqueness using the following procedures. Firstly, the raw scores of three indicators of uniqueness at the provincial level were derived as a function of the number of those with the above-mentioned characteristics divided by the number of users. Secondly, the scores for each

TABLE 2 | Reliability statistics for three uniqueness indicators.

\begin{tabular}{lcc}
\hline & Corrected item-total correlations & Alpha if item deleted \\
\hline PNOT & 0.727 & 0.540 \\
PNE & 0.667 & 0.614 \\
PNFC(R) & 0.638 & 0.852
\end{tabular}

PNOT, percentage of nickname over 10 characters; PNE, percentage of nickname with non-Chinese character; PNFC, percentage of nickname with full Chinese character lower than 5. $R$ indicates the item is reverse scored. Total standardized $\alpha=0.845$. item were standardized across 31 provinces. Thirdly, the overall uniqueness of the nickname score was the sum of individual $(Z)$ scores for the three items. Finally, we transformed the scores by multiplying the means by 20 and then adding 50, to ensure a uniqueness score ranging from approximately 1 to 100 . On this new uniqueness of nickname scale, a higher score represents a higher uniqueness.

As shown in Table 2, the overall standardized alpha for the three-item index was 0.845 . For nicknames with over 10 characters and nicknames with non-Chinese characters, the deletion of these two items would decrease the reliability. But the deletion of nicknames with no more than five Chinese characters or with full Chinese characters would increase the reliability. Furthermore, we included this item for two reasons: (1) more than two items should be included to ensure the reliability of the scale and (2) it is important to expand the scope of uniqueness by nickname and only minimally impact the overall alpha. As for the corrected item total correlations, all items were above 0.400 , recommended by other similar indicator index scales (Yamawaki, 2012). In addition, all the intercorrelations among these three items were moderately and positively correlated as desired, from 0.560 to 0.745 (Table 3). Overall, the three-item index met our statistical criteria of acceptability.

TABLE 3 | Correlation matrix for three uniqueness indicators.

\begin{tabular}{lccc}
\hline & PNOT & PNE & PNFC \\
\hline PNOT & - & - & \\
PNE & $0.745^{\star \star}$ & $0.560^{\star \star}$ & - \\
PNFC(R) & $0.629^{\star \star}$ &
\end{tabular}

PNOT, percentage of nickname over 10 characters; PNE, percentage of nickname with non-Chinese character; PNFC, percentage of nickname with full Chinese character lower than 5. $R$ indicates the item is reverse scored. ${ }^{*} p<0.05$; ${ }^{* *} p<0.01$; ${ }^{* *} p<0.001$. 


\section{PART 2: RELATIONSHIP OF UNIQUENESS WITH CLIMATE}

Having established the reliability and face validity of the uniqueness scale, we then used it to test the main hypothesis of uniqueness and the climato-economic factor in Mainland China. Other factors, such as population density and the percentage of people farming rice/wheat, could also be controlled for.

Climate demand was calculated as the sum of the four absolute deviations from $22^{\circ} \mathrm{C}$ for the average lowest and highest temperatures of the provincial capital in January and July following the method of Van de Vliert et al. (2013).

Income resource was measured by GDP per capita for each province in 2017.

\section{Control Variables}

\section{Urbanization}

The total and urban populations of 2017 were taken from the sixth population census (conducted in 2010) ${ }^{1}$. Urbanization was derived by dividing the urban population by the total population.

\section{Percentage of Populations With College Degree}

The population with college degree of 2017 was taken from the sixth population census (conducted in 2010) (see text footnote 1). The percentage of the population with a college degree was derived by dividing the population with a college degree by the total population.

\section{Population Density}

Population density was taken from the Yearbook of Urban-Rural Development $2017^{2}$.

\section{Percentage of Minorities}

Ethnic population data were taken from the sixth population census (conducted in 2010) (see text footnote 1). The percentage of minorities was derived by dividing all non-Han ethnic population by the total population.

${ }^{1}$ www.stats.gov.cn

${ }^{2}$ www.mohurd.gov.cn

\section{Percentage of Herding Minorities}

Following Talhelm et al. (2014), a few minorities such as the Mongolian Chinese were included as herding minorities. The percentage of minorities was derived by dividing all herding ethnic population by the total population.

\section{Percentage of Cultivated Land Devoted to Rice Paddies}

The data for this variable were extracted from Talhelm et al. (2014).

\section{Results}

We performed all analyses in SPSS 25.0. Xinjiang, Inner Mongolia, Tibet, and Qinghai were excluded for their herding traditions. As Table 4 shows, zero-order correlations between the independent and dependent variables showed that the uniqueness index was positively related to income resource $(r=0.549)$, urbanization $(r=0.615)$, percentage of the population with college degree $(r=0.446)$, and population density $(r=0.665)$ and was negatively correlated to climate demand $(r=-0.278$, n.s.). Although climate demand did not correlate with the uniqueness index significantly, it was also put into the nextregression analysis given the relatively small size $(N=27)$, following Vandello and Cohen's procedure.

We ran a hierarchical regression analysis with the uniqueness index as a dependent variable to explore the main and the interaction effects of climate demand and income resource following Van de Vliert's method. As Table 5 shows, the results in the first step indicates that the population density $(\beta=0.445$, $p=0.071)$ and urbanization $(\beta=1.733, p=0.009)$ had an effect on uniqueness, while the percentage of paddy rice had no effect on uniqueness. In the second step, climate demand $(\beta=-0.425$, $p=0.06)$ and income resource $(\beta=-0.954, p=0.088)$ had a marginally significant effect on uniqueness. The interaction of climate demand and income resource had a marginally significant effect on uniqueness $(\beta=-2.629, p=0.074)$, whereas the main effects of climate demand $(\beta=0.573, p=0.321)$ and income resource $(\beta=1.525, p=0.289)$ were insignificant. However, it was suggested that the interaction effect be excluded due to collinearity issues (variance inflation factor, VIF $=55.5$ ).

TABLE 4 | Correlation matrix for uniqueness index and other variables.

\begin{tabular}{|c|c|c|c|c|c|c|c|c|c|}
\hline & PCS & Urban & PD & PM & PHM & PR & IR & CD & Unique \\
\hline Urban & $0.841^{* \star}$ & & & & & & & & \\
\hline PM & -0.232 & $-0.397^{\star}$ & -0.285 & & & & & & \\
\hline PHM & 0.141 & 0.149 & -0.096 & 0.061 & & & & & \\
\hline $\mathrm{PR}$ & -0.100 & 0.133 & 0.266 & -0.002 & -0.346 & & & & \\
\hline$C D$ & 0.201 & 0.152 & -0.066 & -0.204 & $0.477^{\star}$ & $-0.636^{\star \star}$ & 0.116 & & \\
\hline Unique & $0.446^{\star}$ & $0.615^{\star \star}$ & $0.665^{\star \star}$ & -0.252 & -0.118 & 0.355 & $0.549^{* \star}$ & -0.278 & \\
\hline
\end{tabular}

PCS, percentage of population with college degree; Urban, urbanization; $P D$, population density; IR, GDP per capita; CD, climate demand; PM, percentage of minorities; $P H M$, percentage of herding minorities; $P R$, percentage of paddy rice; Unique, uniqueness indicator; ${ }^{*} p<0.05$, ${ }^{* *} p<0.01 ; N=27$. 
TABLE 5 | Hierarchical regression predicting uniqueness at provincial level.

\begin{tabular}{lccc}
\hline & Model I & Model II & Model III \\
\hline PCS & -0.314 & -0.236 & 0.095 \\
Urban & $1.733^{\star \star}$ & $1.437^{\star}$ & $1.060^{¥}$ \\
PD & $0.445^{¥}$ & $0.470^{¥}$ & 0.321 \\
PM & -0.081 & -0.081 & 0.040 \\
PHM & -0.012 & -0.012 & 0.114 \\
PR & -0.168 & -0.168 & -0.224 \\
IR & & $-0.954^{¥}$ & 1.525 \\
CD & & $-0.425^{¥}$ & 0.573 \\
IR $\times$ CD & & & $-2.629^{\star}$ \\
\hline
\end{tabular}

PCS, percentage of population with college degree; Urban, urbanization; PD, population density; IR, GDP per capita; $C D$, climate demand; PM, percentage of minorities; PHM, percentage of herding minorities; PR, percentage of paddy rice; ${ }^{¥} P<0.10,{ }^{*} p<0.05,{ }^{* *} p<0.01 ; N=27$.

Finally, we ran a regression analysis with the uniqueness index as the dependent variable to explore the main effects of climate demand and income resource. The results showed that climate demand $(\beta=-0.347, p=0.036 ; \beta=-0.425, p=0.060)$ and income resource $(\beta=0.589, p=0.001 ; \beta=-0.954, p=0.088)$ have an effect on the uniqueness of nicknames if other control variables were excluded.

\section{DISCUSSION AND CONCLUSION}

We developed a three-item index, named Chinese uniqueness of nickname index, designed to measure the degree of uniqueness across the provinces of China. Two pieces of evidence were provided to test its validity. Since the three items have face validity, it was obvious that nicknames with over 10 Chinese characters would be considered as more unique than those with three to five Chinese characters. For example, the nickname "meng meng xiao jie shi ge ao jiao de xiao gu liang” (in Chinese, “萌萌小姐是个傲娇的小菇凉”) would be more unique than "wan lai tian yi xue" (in Chinese, “晚来天已雪”). Modernity indexes such as income resource and urbanization were positively related to the uniqueness index at the provincial level.

Our results also lent partial support to the climato-economic theory of collectivism. Harsh climates may encourage inhabitants to favor group harmony and avoid being different from group members to cope with bad environment (Van de Vliert, 2013). When people from these areas choose names for themselves on social media, they prefer to use common names, which results in a lower uniqueness at the provincial level. Although livability has improved with the advent of modernization, climate demand remains imprinted on the inhabitants' variations on uniqueness.

Income resource was not supported in this study. At first glance, income resource predicted uniqueness as more income resource meant that the inhabitants were more likely to select a unique nickname on social media. However, this is in contrast to the previous finding in the Chinese mainland. The previous study found that income resource would moderate the effect of climate demand on collectivism as inhabitants of those areas with harsh climatic demand and higher income resource would be least collectivistic (Van de Vliert et al., 2013). This implies that such inhabitants would select a more unique nickname. Some factors may explain this inconsistency. Firstly, income resource is the secondary factor in the climatoeconomic theory of collectivism, in which the interaction with climate demand was more important than its main effect (Van de Vliert, 2013, p. 507). Unfortunately, due to the small size of the provinces and collinearity, the interaction effect could not be used to support the climato-economic theory. Secondly, the main effect of income resource on collectivism is not robust. Even in their study in China, the main effect of income resource was not always significant on collectivism (Van de Vliert et al., 2013; Table 4). Thirdly, income resource was just one of the resources that could be used to cope with hot or cold climate stress. Other resources such as geographical resources could be explored in future studies.

Our findings make theoretical contributions to both the climato-economic theory of culture and computational social psychology. Firstly, it enriches the climato-economic theory of collectivism by exploring its effect on real behavior in OSNs. In this series of studies on the climato-economic theory of collectivism, Van de Vliert relied heavily on secondary data and the self-report measure of collectivism (Van de Vliert, 2007, 2011, 2013). While such rating scales are good at assessing individual differences within a group, they should be complemented by other measures such as cultural products or cognitive tasks (Chen and Kitayama, 2013). Our results found that climate demand could at least predict the uniqueness in nickname preference. Inhabitants in provinces with harsh climatic demands are less likely to make up unique nicknames than those from provinces with comfortable climate demands. This result highlighted its generalizability not only on psychological traits but also on behavior in real settings.

The study also has contribution to computational social psychology. Big data presents unprecedented opportunities to understand human behavior on a large scale. Data and computer scientists often use bottom-up, data-driven methods to predict social psychological variables, such as personality traits, by Facebook likes (Youyou et al., 2015) or just algorithms from machine learning based on the digital records of users (Kosinski et al., 2013). This makes it difficult to elucidate without other evidence. There is a possible way to solve this problem of big data as including theoretically relevant variables as predictors could complement any shortcoming of the data-driven method (Qiu et al., 2018). Our study attempted to combine a data-driven explanation with a theoretical one. Firstly, we conceptualized the uniqueness index theoretically and then found that climate demand could explain provincial variations on uniqueness. It helped to provide a meaningful explanation of social psychological phenomena with big data and avoid mere predictions (Qiu et al., 2018).

Our findings also lend support to the advantage of intranational-level unit analysis in the link between ecology and 
cultural values (Vandello and Cohen, 1999). Compared with national-level unit analysis, intranational-level unit analysis (such as provinces in China or states in the United States) within one culture or nation reduces criticisms based on language, religions, measurement errors for large nations, sociological indicators of collectivism, and psychometric equivalence across distinct populations (Van de Vliert et al., 2013). In our study, other confounding factors such as language and religion were well controlled for. The overwhelming majority of Sina Weibo users would be Han Chinese. Increasingly, studies have combined national and intranational comparisons to explore the relationship between cultural values and ecological factors (Thomson et al., 2018).

China is a natural and ideal cultural entity to explore the relationship between cultural values and ecological factors. On the one hand, from the geographical and ecological perspectives, the range within China is similar to that in Europe and the United States. Until now, subsistence-style work such as herding and farming rice/wheat had been proven using a Chinese sample (Chua et al., 2019; Stojcic et al., 2020). On the other hand, since China has been keeping records and maintaining databases for over 2,000 years, this could be used to explore the origin of cultural values with spatiotemporal evidence in the future.

Our results did not support the rice theory of collectivism. Farming rice/wheat has been found in the Chinese mainland to predict that inhabitants in rice-farming provinces would be more likely to be collectivistic than those in wheat-farming provinces (Talhelm et al., 2014; Dong et al., 2019). Our results, however, showed that the percentage of paddy rice did not predict uniqueness. We put aside the significance of the coefficient that Sina Weibo users from rice-farming provinces were more likely to give themselves unique nicknames than those from provinces farming wheat, which is indicative of the opposite of the collectivistic theory.

Collectivism is a loosely connected concept in which different facets may be sensitive to different ecological factors. For instance, in-group favoritism and out-group exclusion may be more sensitive than holistic thinking to the perceived threat of infectious diseases (Fincher et al., 2008). In this study, uniqueness may be sensitive toward the climate demand, but not to farming rice/wheat.

Population density was found to predict uniqueness positively: inhabitants in provinces with a higher population density prefer more unique nicknames than those in lower ones. This was consistent with the hypothesis that a higher population density is associated with a stronger collectivism (Vandello and Cohen, 1999; Gelfand et al., 2011). Within China, population density was highly correlated with the modernity indicators such as urbanization and GDP per capita. Modernity predicted uniqueness positively. The effect of population density on uniqueness probably was reverted or offset by modernity.

We also considered some limitations. Firstly, some authors have argued that intra-provincial variances should not be neglected, especially the relationship between ecological factors and collectivism (Ren et al., 2021). We avoided it because not enough ecological data were available at the city or the county level. It could be improved in the future to explore this topic at the city level, when available. Secondly, three factors were included in this study. Other factors such as infectious diseases could also be explored to make the results robust. Thirdly, location labels reported by the users themselves were thought to be the actual provinces where they grew up. It was impossible to guarantee the accuracy of the classification. However, we do not believe that this would pose a significant problem. As most people report their actual location on social network platforms, we are open to new methods and research to find a better way to obtain users' locations in order to explore whether or not our finding is robust.

Overall, based on Sina Weibo users' nicknames, we constructed a uniqueness index with reliability and face validity. Furthermore, the study also found that climate demand could explain and predict uniqueness. Harsh climate could make inhabitants unlikely to use unique nicknames on online social platforms.

\section{DATA AVAILABILITY STATEMENT}

The raw data supporting the conclusions of this article will be made available by the authors, without undue reservation.

\section{ETHICS STATEMENT}

This study involving human participants were reviewed and approved by the Institutional Review Board of the Institute of Psychology, Chinese Academy of Sciences. Written informed consent from the participants was not required to participate in this study in accordance with the national legislation and the institutional requirements because our data was collected from publicly available information on an open online social networking site through Weibo's application programming interface.

\section{AUTHOR CONTRIBUTIONS}

XR: conception and design of the study. LH and YC: acquisition of the data. XR and LH: analysis and interpretation of the data and drafting the manuscript. XR, LH, and YC: revising the manuscript critically for important intellectual content. All authors contributed to the article and approved the submitted version.

\section{FUNDING}

This paper was funded by National Social Science Fund of China project "the effect of COVID-19 on ingroup favoritism/outgroup exclusion and its mechanism" (20BSH142). 


\section{REFERENCES}

Back, M. D., Stopfer, J. M., Vazire, S., Gaddis, S., Schmukle, S. C., Egloff, B., et al. (2010). Facebook profiles reflect actual personality, not self-idealization. Psychol. Sci. 21, 372-374. doi: 10.1177/0956797609360756

Bazzi, S., Fiszbein, M., and Gebresilasse, M. (2020). Frontier culture: The roots and persistence of "rugged individualism" in the United States. Econometrica, 88, 2329-2368. doi: 10.3982/ECTA16484

Chen, D., and Kitayama, S. (2013). Frontier migration fosters ethos of independence: deconstructing the climato-economic theory of human culture. Behav. Brain Sci. 36, 486-487. doi: 10.1017/s0140525×13000083

Chen, X., Ren, X. P., and Zhang, X. K. (2019). Shenzhen spirit fosters independent agency. Chin. Soc. Psychol. Rev. 16, 14-37.

Chua, R. Y. J., Huang, K. G., and Jin, M. (2019). Mapping cultural tightness and its links to innovation, urbanization, and happiness across 31 provinces in China. Proc. Natl. Acad. Sci. U.S.A. 116, 6720-6725. doi: 10.1073/pnas. 1815723116

Dong, X., Talhelm, T., and Ren, X. (2019). Teens in rice county are more interdependent and think more holistically than nearby wheat county. Soc. Psychol. Pers. Sci. 10, 966-976. doi: 10.1177/1948550618808868

Fincher, C. L., Thornhill, R., Murray, D. R., and Schaller, M. (2008). Pathogen prevalence predicts human cross-cultural variability in individualism/collectivism. Proc. R. Soc. B Biol. Sci. 275, 1279-1285. doi: $10.1098 /$ rspb.2008.0094

Fischer, R., and Van de Vliert, E. (2011). Does climate undermine subjective wellbeing? A 58-nation study. Pers. Soc. Psychol. Bull. 37, 1031-1041. doi: 10.1177/ 0146167211407075

Gelfand, M. J., Raver, J. L., Nishii, L., Leslie, L. M., Lun, J., Lim, B. C., et al. (2011). Differences between tight and loose cultures: a 33-nation study. Science 332, 1100-1104.

Hou, D. X., Ren, X. P., and Zhang, F. (2016). China's collectivism scale by objective indicator. Chin. Soc. Psychol. Rev. 11, 86-98.

Kim, H., and Markus, H. R. (1999). Deviance or uniqueness, harmony or conformity? A cultural analysis. J. Pers. Soc. Psychol. 77, 785-800. doi: 10.1037/ 0022-3514.77.4.785

Kosinski, M., Stillwell, D., and Graepel, T. (2013). Private traits and attributes are predictable from digital records of human behavior. Proc. Natl. Acad. Sci. U.S.A. 110, 5802-5805. doi: 10.1073/pnas. 1218772110

Liu, S. S., Morris, M. W., Talhelm, T., and Yang, Q. (2019). Ingroup vigilance in collectivistic cultures. Proc. Natl. Acad. Sci. U.S.A. 116, 14538-14546. doi 10.1073/pnas. 1817588116

Na, J., Grossmann, I., Varnum, M. E. W., Karasawa, M., Cho, Y., Kitayama, S., et al. (2020). Culture and personality revisited: behavioral profiles and withinperson stability in interdependent (vs. independent) social orientation and holistic (vs. analytic) cognitive style. J. Pers. 88, 908-924. doi: 10.1111/jopy. 12536

Oyserman, D. (2017). Culture three ways: culture and subcultures within countries. Annu. Rev. Psychol. 68, 435-463. doi: 10.1146/annurev-psych-122414-033617

Oyserman, D., Coon, H. M., and Kemmelmeier, M. (2002). Rethinking individualism and collectivism: evaluation of theoretical assumptions and meta-analyses. Psychol. Bull. 128, 3-72. doi: 10.1037/0033-2909.128.1.3

Qiu, L., Chan, S. H. M., and Chan, D. (2018). Big data in social and psychological science: theoretical and methodological issues. J. Comput. Soc. Sci. 1, 59-66. doi: 10.1007/s42001-017-0013-6
Ren, X., Cang, X., and Ryder, A. G. (2021). An integrated ecological approach to mapping variations in collectivism within China: introducing the triple-line framework. J. Pac. Rim Psychol. 15, 1-12.

Statista (2020). China: Number of Social Network Users 2017-2025. Hamberg: Statista.

Stojcic, I., Dong, X., and Ren, X. (2020). Body image and sociocultural predictors of body image dissatisfaction in croatian and Chinese women. Front. Psychol. 11:731. doi: 10.3389/fpsyg.2020.00731

Talhelm, T., Zhang, X., Oishi, S., Shimin, C., Duan, D., Lan, X., et al. (2014). Largescale psychological differences within China explained by rice versus wheat agriculture. Science 344, 603-608. doi: 10.1126/science.1246850

Thomson, R., Yuki, M., Talhelm, T., Schug, J., Kito, M., Ayanian, A. H., et al. (2018). Relational mobility predicts social behaviors in 39 countries and is tied to historical farming and threat. Proc. Natl. Acad. Sci. U.S.A. 115, 7521-7526. doi: 10.1073/pnas.1713191115

Twenge, J. M., Abebe, E. M., and Campbell, W. K. (2010). "Fitting in or standing out: trends in American parents' choices for Children's names, 1880-2007. Soc. Psychol. Pers. Sci. 1, 19-25. doi: 10.1177/1948550609349515

Van de Vliert, E. (2007). Climatoeconomic roots of survival versus self-expression cultures. J. Cross Cult. Psychol. 38, 156-172. doi: 10.1177/0022022106297298

Van de Vliert, E. (2011). Climato-economic origins of variation in ingroup favoritism. J. Cross Cult. Psychol. 42, 494-515. doi: 10.1177/002202211038 1120

Van de Vliert, E. (2013). Climato-economic habitats support patterns of human needs, stresses, and freedoms. Behav. Brain Sci. 36, 465-480. doi: 10.1017/ s0140525x12002828

Van de Vliert, E. (2020). The global ecology of differentiation between us and them. Nat. Hum. Behav. 4, 270-278. doi: 10.1038/s41562-019-0783-3

Van de Vliert, E., Yang, H., Wang, Y., and Ren, X.-P. (2013). Climato-economic imprints on Chinese collectivism. J. Cross Cult. Psychol. 44, 589-605. doi: $10.1177 / 0022022112463605$

Vandello, J. A., and Cohen, D. (1999). Patterns of individualism and collectivism across the United States. J. Pers. Soc. Psychol. 77, 279-292. doi: 10.1037/00223514.77.2.279

Varnum, E. W., and Kitayama, S. (2011). Whats in a name: Popular names are less common on frontiers. Psychol. Sci. 22:176. doi: 10.1177/0956797610395396

World Bank (2020). List of Countries by GDP (nominal) Per Capita. Washington, DC: Worldbank.

Yamawaki, N. (2012). Within-culture variations of collectivism in Japan. J. Cross Cult. Psychol. 43, 1191-1204. doi: 10.1177/0022022111428171

Youyou, W., Kosinski, M., and Stillwell, D. (2015). Computer-based personality judgments are more accurate than those made by humans. Proc. Natl. Acad. Sci. U.S.A. 112, 1036-1040. doi: 10.1073/pnas. 1418680112

Conflict of Interest: The authors declare that the research was conducted in the absence of any commercial or financial relationships that could be construed as a potential conflict of interest.

Copyright (C) $2021 \mathrm{He}$, Chen and Ren. This is an open-access article distributed under the terms of the Creative Commons Attribution License (CC BY). The use, distribution or reproduction in other forums is permitted, provided the original author(s) and the copyright owner(s) are credited and that the original publication in this journal is cited, in accordance with accepted academic practice. No use, distribution or reproduction is permitted which does not comply with these terms. 